\title{
HADAMARD DUALS, RETRACTABILITY AND OPPENHEIM'S INEQUALITY
}

\author{
Shaun M. FALlat AND CHARLES R. JOHNSON
}

\begin{abstract}
Oppenheim's determinantal inequality was originally proved for positive semidefinite matrices and has produced many interesting consequences and applications. Positive semidefinite matrices were a natural class to consider partly because they are closed under Hadamard (or entry-wise) multiplication. Since Oppenheim's original contribution, others have considered similar inequalities for $M$-matrices, inverse $M$-matrices and totally nonnegative matrices. We attempt to unify many of these existing results dealing with Oppenheim's inequality, and our approach relies on two major themes: retractions and Hadamard duals. Retractions are a type of diagonal perturbation and the Hadamard dual is a maximal collection of matrices with a closure property under Hadamard multiplication. These notions are applied to yield results that generalize Oppenheim's original result.
\end{abstract}

\section{Mathematics subject classification (2000): 15A48, 15A57.}

Key words and phrases: Hadamard products, $P$-matrices, positive semidefinite matrices, $M$-matrices, inverse $M$-matrices, Oppenheim's inequality, totally nonnegative matrices, retractability, determinantal inequalities.

\section{REFERENCES}

[1] B. A. Asner, On the Total Nonnegativity of the Hurwitz Matrix, SIAM J. Appl. Math. 18 No. 2 (1970) 407-414.

[2] D. CARLSON, Weakly, Sign-Symmetric Matrices and Some Determinantal Inequalities, Colloq. Math. 17 (1967) 123-129.

[3] S. CHEN, A property concerning the Hadamard powers of inverse $M$-matrices, Linear Algebra Appl., 381 (2004) 53-60.

[4] A. S. Crans, S. M. Fallat and C. R. Johnson, The Hadamard core of the totally nonnegative matrices, Linear Algebra Appl., 328 (2001) 203-222.

[5] S. M. FALLAT, Bidiagonal Factorizations of Totally Nonnegative Matrices, American Math. Monthly 109 (2001) 697-712.

[6] S. M. Fallat and C. R. Johnson, Sub-direct Sums and Positivity Classes of Matrices, Linear Algebra Appl. 288 (1999) 149-173.

[7] S. M. Fallat, C. R. Johnson and R. L. Smith, The General Totally Positive Matrix Completion Problem with Few Unspecified Entries, Electron. J. Linear Algebra, 7 (2000) 1-20.

[8] J. GARLOFF AND D. G. WAGNER, Hadamard Products of Stable Polynomials are Stable, J. Math. Anal. Appl. 202 (1996) 797-809.

[9] R. A. HoRn, The Hadamard Product, in Matrix Theory and Applications, C. R. Johnson, ed., Proc. Sympos. Appl. Math., 40 (1089) 87-169.

[10] R. A. Horn And C. R. Johnson, Matrix Analysis, Cambridge University Press, New York, 1985.

[11] R. A. HoRn AND C. R. Johnson, Topics in Matrix Analysis, Cambridge University Press, New York, 1991.

[12] C. R. Johnson, A Hadamard Product Involving M -Matrices, Linear and Multilinear Algebra, 4 (1977) 261-264.

[13] C. R. Johnson, Inverse M-matrices, Linear Algebra Appl. 47 (1982) 195-216. 
[14] C. R. Johnson, Closure Properties of Certain Positivity Classes of Matrices under Various Algebraic Operations, Linear Algebra Appl. 97 (1987) 243-247.

[15] C. R. Johnson AND R. L. SMITH, Path Product Matrices, Linear and Multilinear Algebra, 46 (1999) $177-191$.

[16] C. R. JoHNSON AND R. L. SMITH, Path Product matrices and Eventually Inverse M-matrices, to appear in SIAM Journal on Matrix Anal. Appl.

[17] C. R. Johnson And R. L. Smith, Positive, Path Product, and Inverse M-matrices, to appear in Linear Algebra Appl.

[18] C. R. JohNSON AND R. L. SMITH, Monotonicity of Continuous Hadamard Powers of Inverse M-matrices, to be submitted.

[19] D. G. Wagner, Total Positivity of Hadamard Products, J. Math. Anal. Appl. 163 (1992) 459-483.

[20] B-Y. WANG, X. ZHANG AND F. ZHANG, On the Hadamard product of inverse $M$-matrices, Linear Algebra Appl. 305 (2000) 23-31. 